\title{
ADAPTIVE CONTROL OF A TWO INPUT - TWO OUTPUT SYSTEM
}

\author{
Marek Kubalčik, Vladimír Bobál \\ Department of Control Theory, Institute of Information Technologies \\ Tomas Bata University \\ Nám. TGM 275, 76272 Zlín, Czech Republic \\ Phone, Fax:+420677543217,E-mail:kubalcik@ft.utb.cz
}

\begin{abstract}
This paper presents the design and simulation of adaptive control for a two input-two output system together with the real-time control of a laboratory model using this design method. The synthesis is based on a polynomial approach. Decoupling, where the compensator is placed ahead of the system, suppresses the interactions between control loops. The results of the simulation and the real-time experiments are also given. Copyright 2001 IFAC
\end{abstract}

Keywords: multivariable control, control algorithms, decoupling, compensators, adaptive control

\section{INTRODUCTION}

Many technological processes require that several variables relating to one system are controlled simultaneously. Each input may influence all system outputs. The design of a controller able to cope with such a system must be quite sophisticated. There are many different methods of controlling multivariable systems. Several of these use decentralized PID controllers (Luyben 1986), others apply single inputsingle-output (SISO) methods extended to cover multiple inputs (Chien et al. 1987). Here decoupling methods are used to transform the multivariable system into a series of independent SISO loops (Krishnawamy et al. 1991; Tade et al. 1986; Wittenmark el al. 1987; Skogestad and Postlethwaite 1996).

This paper is organized as follows: Section 2 presents the controlled model; Section 3 describes how feedback control without decoupling is designed; Section 4 describes two decoupling methods; Section 5 describes the system identification method; Section 6 gives the simulation results; Section 7 contains the experimental results; finally, Section 8 concludes the paper.

\section{A DESCRIPTION OF A TWO INPUT - TWO OUTPUT SYSTEM}

The internal structure of the the system is shown in Fig. I

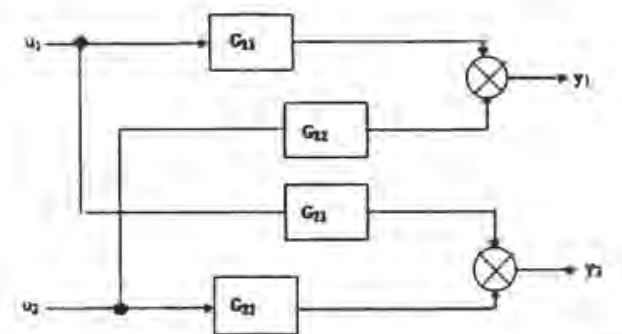

Fig. 1. A two input - two output system - the "P" structure

The transfer matrix of the system is

$$
\boldsymbol{G}=\left[\begin{array}{ll}
G_{11} & G_{12} \\
G_{21} & G_{22}
\end{array}\right]
$$

It is possible to assume that the system is described by the matrix fraction

$$
G\left(z^{-1}\right)=A^{-1}\left(z^{-1}\right) B\left(z^{-1}\right)=B_{1}\left(z^{-1}\right) A_{1}^{-1}\left(z^{-1}\right)
$$


Where polynomial matrices $A \in R_{m m}\left[z^{-1}\right], B \in R_{m n}\left[z^{-1}\right]$ are the left indivisible decomposition of matrix $\boldsymbol{G}\left(z^{-1}\right)$ and matrices $A_{1} \in R_{m m}\left[z^{-1}\right], B_{1} \in R_{m n}\left[z^{-1}\right]$ are the right indivisible decomposition.

The matrices of the discrete model are

$$
\begin{gathered}
A\left(z^{-1}\right)=\left[\begin{array}{cc}
1+a_{1} z^{-1}+a_{2} z^{-2} & a_{3} z^{-1}+a_{4} z^{-2} \\
a_{5} z^{-1}+a_{6} z^{-2} & 1+a_{7} z^{-1}+a_{8} z^{-2}
\end{array}\right] \\
B\left(z^{-1}\right)=\left[\begin{array}{ll}
b_{1} z^{-1}+b_{2} z^{-2} & b_{3} z^{-1}+b_{4} z^{-2} \\
b_{5} z^{-1}+b_{6} z^{-2} & b_{7} z^{-1}+b_{8} z^{-2}
\end{array}\right]
\end{gathered}
$$

and the differential equations of the model are

$$
\begin{aligned}
& y_{1}(k)=-a_{1} y_{1}(k-1)-a_{2} y_{1}(k-2)-a_{3} y_{2}(k-1)- \\
& -a_{4} y_{2}(k-2)+b_{1} u_{1}(k-1)+b_{2} u_{1}(k-2)+ \\
& +b_{3} u_{2}(k-1)+b_{4} u_{2}(k-2) \\
& y_{2}(k)=-a_{5} y_{1}(k-1)-a_{6} y_{1}(k-2)-a_{7} y_{2}(k-1)- \\
& -a_{8} y_{2}(k-2)+b_{5} u_{1}(k-1)+b_{6} u_{1}(k-2)+ \\
& +b_{7} u_{2}(k-1)+b_{8} u_{2}(k-2)
\end{aligned}
$$

\section{DESIGNING FEEDBACK CONTROL}

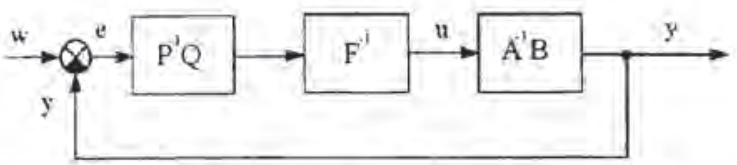

Fig. 2. Block diagram of the closed loop system

In the same way as the controlled system, the transfer matrix of the controller takes the form of matrix fraction

$$
\boldsymbol{G}\left(z^{-1}\right)=\boldsymbol{P}^{-1}\left(z^{-1}\right) \boldsymbol{Q}\left(z^{-1}\right)=\boldsymbol{Q}_{1}\left(z^{-1}\right) \boldsymbol{P}_{1}^{-1}\left(z^{-1}\right)
$$

The matrix of an integrator for permanent zero control error is

$$
F\left(z^{-1}\right)=\left[\begin{array}{cc}
1-z^{-1} & 0 \\
0 & 1-z^{-1}
\end{array}\right]
$$

The control law apparent in the block diagram (operator $z^{-1}$ will be omitted from some operations for the sake of simplification) has the form

$$
\boldsymbol{U}=\boldsymbol{F}^{-1} \boldsymbol{Q}_{1} \boldsymbol{P}_{1}^{-1} \boldsymbol{E}
$$

It is possible to derive the following equation for the system output

$$
\boldsymbol{Y}=A^{-1} B F^{-1} P^{-1} Q E=A^{-1} B F^{-1} P^{-1} Q(W-Y)
$$

which can be modified to give

$$
Y=P_{1}\left(A F P_{1}+B Q_{l}\right)^{-1} B Q_{1} P_{1}^{-1} W
$$

The closed loop system is stable when the following diophantine equation is satisfied

$$
A F P_{1}+B Q_{1}=M
$$

where $\boldsymbol{M}\left(z^{-1}\right) \in R_{m m}\left[z^{-1}\right]$ is a stable diagonal polynomial matrix.

$$
M\left(z^{-1}\right)=\left[\begin{array}{cc}
1+m_{1} z^{-1}+m_{2} z^{-2}+ & 0 \\
+m_{3} z^{-3}+m_{4} z^{-4} & 1+m_{5} z^{-1}+m_{6} z^{-2}+ \\
0 & +m_{7} z^{-3}+m_{8} z^{-4}
\end{array}\right]
$$

The roots of this polynomial matrix are the ruling factor in the behaviour of the closed loop system. They must be inside the unit circle if the system is to be stable.

The degree of the controller matrices polynomials depends on the internal properness of the closed loop. The structure of matrices $\boldsymbol{P}_{1}$ and $\boldsymbol{Q}_{1}$ was chosen so that the number of unknown controller parameters equals the number of algebraic equations resulting from the solution of the diophantine equations using the uncertain coefficients method.

$$
\begin{gathered}
P_{1}\left(z^{-1}\right)=\left[\begin{array}{cc}
1+p_{1} z^{-1} & p_{2} z^{-1} \\
p_{3} z^{-1} & 1+p_{4} z^{-1}
\end{array}\right] \\
\boldsymbol{Q}_{1}\left(z^{-1}\right)=\left[\begin{array}{cc}
q_{1}+q_{2} z^{-1}+q_{3} z^{-2} & q_{4}+q_{5} z^{-1}+q_{6} z^{-2} \\
q_{7}+q_{8} z^{-1}+q_{9} z^{-2} & q_{10}+q_{11} z^{-1}+q_{12} z^{-2}
\end{array}\right]
\end{gathered}
$$

The solution to the diophantine equation results in a set of sixteen algebraic equations with unknown controller parameters. The controller parameters are given by solving these equations.

\section{DESIGNING DECOUPLING CONTROL USING COMPENSATORS}

There are several ways to control multivariable systems with internal interactions. Some make use of decentralized PID controllers, whilst others are composed of a string of single input - single output methods.

One possibility is the serial insertion of a compensator ahead of the system (Krishnawamy et al. 1991; Peng 1990; Tade et al. 1986; Wittenmark et al. 1987). The aim here is to suppress of undesirable interactions between the input and output variables so that each input affects only one controlled variable.

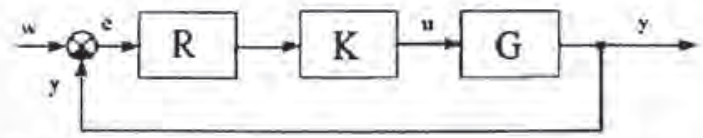

Fig. 3. A Closed loop system with compensator

The resulting transfer function $\boldsymbol{H}$ is then given by

$$
\boldsymbol{H}=\boldsymbol{K} \boldsymbol{G}
$$

The decoupling conditions are fulfilled when matrix $\boldsymbol{H}$ is diagonal.

Several well - known compensators are given in (Krishnawamy et al. 1991; Peng 1990; Tade et al. 
1986; Wittenmark et al. 1987). Control algorithms were derived for the model above with two compensators. These will be referred to as $\mathbf{C}_{1}$ and $\mathbf{C}_{2}$.

Compensator $C_{1}$ is the inversion of the controlled system. Matrix $\boldsymbol{H}$ is, therefore, a unit matrix.

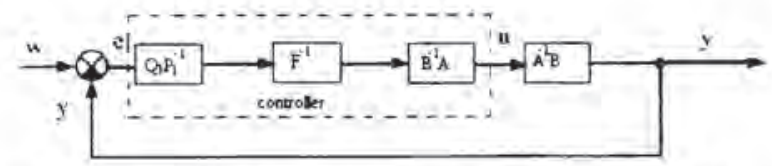

Fig. 4. The closed loop system with compensator $C_{1}$

This block diagram leads to an equation for the system output which takes the form

$$
\boldsymbol{Y}=\boldsymbol{P}_{l}\left(F P_{1}+Q_{l}\right)^{-1} Q_{1} P_{l}^{-1} W
$$

The following equation must be satisfied if the closed loop system is to be stable

$$
\boldsymbol{F} \boldsymbol{P}_{1}+\boldsymbol{Q}_{1}=\boldsymbol{M}
$$

The structure of the polynomial matrices of the controller were chosen to suit physical demands.

$$
\begin{gathered}
\boldsymbol{P}_{1}\left(z^{-1}\right)=\left[\begin{array}{ll}
1 & 0 \\
0 & 1
\end{array}\right] \\
\boldsymbol{Q}_{1}\left(z^{-1}\right)=\left[\begin{array}{cc}
q_{1} z^{-1} & 0 \\
0 & q_{2} z^{-1}
\end{array}\right]
\end{gathered}
$$

Consequently, matrix $\boldsymbol{M}$ was chosen to be

$$
\boldsymbol{M}\left(z^{-1}\right)=\left[\begin{array}{cc}
1+m_{1} z^{-1} & 0 \\
0 & 1+m_{2} z^{-1}
\end{array}\right]
$$

The controller parameters are the result from the equation (15). The control law can be described by matrix equation

$$
F U=B^{-1} A Q_{1} P_{1}^{-1} E
$$

Compensator $\mathbf{C}_{2}$ is adjugated matrix $\boldsymbol{B}$. When $\mathbf{C}_{2}$ was included in the design of the closed loop the model was simplified by considering matrix $\boldsymbol{A}$ as diagonal. The multiplication of matrix $\boldsymbol{B}$ and adjugated matrix $\boldsymbol{B}$ results in diagonal matrix $\boldsymbol{H}$. The determinants of matrix $B$ represent the diagonal elements. When matrix $A$ is nondiagonal, its inverted form must be placed ahead of the system in order to obtain diagonal matrix $\boldsymbol{H}$, otherwise it may increase the order of the controller and sophistication of the closed loop system. Although designed for a diagonal matrix, compensator $C_{2}$ also improves the control process for non - diagonal matrix $\boldsymbol{A}$ in the controlled system. This is demonstrated in the simulation results.

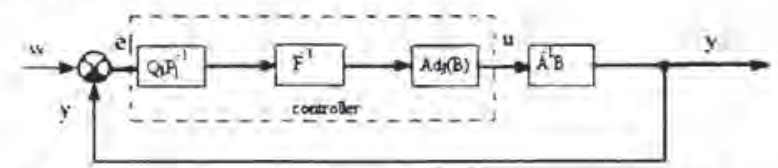

Fig. 5. The closed loop system with compensator $\mathbf{C}_{2}$

The equation for the system output as shown in this block diagram takes the form

$$
\boldsymbol{Y}=\boldsymbol{P}_{1}\left(A F P_{1}+B_{v} Q_{1}\right) B_{v} Q_{1} P_{1}^{-1} W
$$

where

$$
\boldsymbol{B}_{v}=\operatorname{Badj}(\boldsymbol{B})=\left[\begin{array}{cc}
\operatorname{det}(\boldsymbol{B}) & 0 \\
0 & \operatorname{det}(\boldsymbol{B})
\end{array}\right]
$$

To achieve stability in the closed loop system the following diophantine equation must be fulfilled

$$
A F P_{1}+B_{v} Q_{1}=M
$$

The controller polynomial matrices are chosen as shown below

$$
\begin{gathered}
\boldsymbol{P}_{1}\left(z^{-1}\right)=\left[\begin{array}{cc}
1+p_{1} z^{-1} & p_{2} z^{-1} \\
p_{3} z^{-1} & 1+p_{4} z^{-1}
\end{array}\right] \\
\boldsymbol{Q}_{1}\left(z^{-1}\right)=\left[\begin{array}{cc}
q_{1}+q_{2} z^{-1}+q_{3} z^{-2} & q_{4}+q_{5} z^{-1}+q_{6} z^{-2} \\
q_{7}+q_{8} z^{-1}+q_{9} z^{-2} & q_{10}+q_{11} z^{-1}+q_{12} z^{-2}
\end{array}\right]
\end{gathered}
$$

and matrix $\boldsymbol{M}$ is

$$
M\left(z^{-1}\right)=\left[\begin{array}{cc}
1+m_{1} z^{-1}+ & \\
+m_{2} z^{-2}+m_{3} z^{-3}+ & 0 \\
+m_{4} z^{-4}+m_{5} z^{-5} & \\
0 & \begin{array}{l}
1+m_{6} z^{-1}+ \\
m_{7} z^{-2}+m_{4} z^{-3}+ \\
\end{array} \\
& +m_{9} z^{-4}+m_{10} z^{-5}
\end{array}\right]
$$

Solving the diophantine equation defines a set of algebraic equations which we subsequently use to obtain the unknown controller parameters.

The control law is given by the block diagram

$$
\boldsymbol{F} \boldsymbol{U}=\operatorname{adj}(\boldsymbol{B}) \boldsymbol{Q}_{1} \boldsymbol{P}_{1}^{-1} \boldsymbol{E}
$$

\section{IDENTIFICATION}

The algorithms designed here were incorporated into an adaptive control system with recursive identification. The recursive least squares method proved effective for self-tuning controllers (Bittanti et al. 1990) and was used as the basis for our algorithm. For our two-variable example we considered the disintegration of identification into two independent parts.

The parameter vectors are completed as shown below:

$$
\begin{aligned}
\boldsymbol{\theta}_{1}^{T}(k) & =\left[a_{1}, a_{2}, a_{3}, a_{4}, b_{1}, b_{2}, b_{3}, b_{4}\right] \\
\boldsymbol{\theta}_{2}{ }^{T}(k) & =\left[a_{5}, a_{6}, a_{7}, a_{8}, b_{5}, b_{6}, b_{7}, b_{8}\right]
\end{aligned}
$$

The data vector is

$$
\begin{aligned}
& \phi^{T}(k-1)=\left[-y_{1}(k-1),-y_{1}(k-2),-y_{2}(k-1),\right. \\
& \left.-y_{2}(k-2), u_{1}(k-1), u_{1}(k-2), u_{2}(k-1), u_{2}(k-2)\right]
\end{aligned}
$$

The parameter estimates are actualized using the recursive least squares method plus directional forgetting. 


\section{SIMULATION}

Matlab + Simulink for Windows (The MathWork, Inc.) were used to create a program and diagrams to simulate and verify the algorithms. Verification by simulation was carried out on a range of systems with varying dynamics. The control of the model below is given here as our example.

$$
\begin{gathered}
A\left(z^{-1}\right)=\left[\begin{array}{cc}
1+0.3 z^{-1}+0,1 z^{-2} & 0.1 z^{-1}+0.2 z^{-2} \\
0.1 z^{-1}+0,3 z^{-2} & 1+0,3 z^{-1}+0,1 z^{-2}
\end{array}\right] \\
B\left(z^{-1}\right)=\left[\begin{array}{cc}
0,1 z^{-1}+0,4 z^{-2} & 0.9 z^{-1}+0.4 z^{-2} \\
0.6 z^{-1}+0,2 z^{-2} & 0.3 z^{-1}+0.4 z^{-2}
\end{array}\right]
\end{gathered}
$$

Fig. 6 shows the system's step response

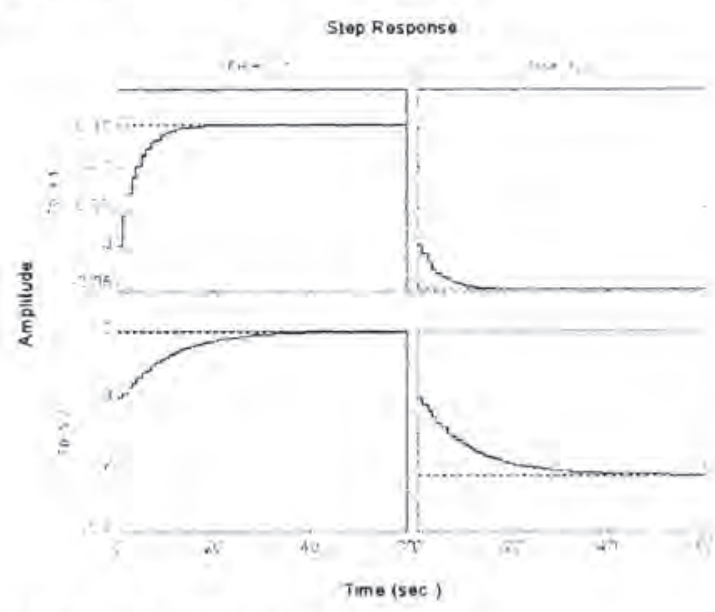

Fig. 6. The step response of the system

The right side control matrices are denoted as follows: without compensator - $\boldsymbol{M}_{1}$, with compensator $\mathbf{C}_{\mathbf{I}}-\boldsymbol{M}_{2}$, and with compensator $\mathbf{C}_{\mathbf{2}}-\boldsymbol{M}_{3}$.

$$
\begin{gathered}
M_{1}\left(z^{-1}\right)=\left[\begin{array}{cc}
\left(1-0,1 z^{-1}\right)^{4} & 0 \\
0 & \left(1-0,1 z^{-1}\right)^{4}
\end{array}\right] \\
M_{2}\left(z^{-1}\right)=\left[\begin{array}{cc}
1-0,1 z^{-1} & 0 \\
0 & 1-0,1 z^{-1}
\end{array}\right] \\
M_{3}\left(z^{-1}\right)=\left[\begin{array}{cc}
\left(1-0,1 z^{-1}\right)^{5} & 0 \\
0 & \left(1-0,1 z^{-1}\right)^{5}
\end{array}\right]
\end{gathered}
$$

The same initial conditions for system identification were used for all the types of adaptive control we tested. The initial parameter estimates were chosen to be

$$
\begin{aligned}
& \boldsymbol{\theta}_{1}{ }^{\top}(0)=[0.1,0.2 .0 .3,0.4,0.1,0.2,0.3,0.4] \\
& \boldsymbol{\theta}_{2}{ }^{\top}(0)=[0.5,0.6,0.7,0.8,0.5,0.6,0.7,0.8]
\end{aligned}
$$

The results of simulation are shown in Figs 7-12 .

It is possible to draw several conclusions from the simulation results of the experiments on linear static systems. The basic requirement to ensure permanent zero control error was satisfied in all cases. The criteria on which we judge the quality of the control process are the overshoot on the controlled values and the speed with which zero control error is achieved. According to these criteria the controller incorporating compensator $\boldsymbol{C}_{1}$ performed the best. However, this controller appears to be unsuited to adaptive control due to the size of the overshoot and the large numbers of process and controller outputs. The controller which uses compensator $\mathbf{C}_{2}$ seems to work best in adaptive control. With regards to decoupling, it is clear that controllers with compensators greatly reduce interaction.
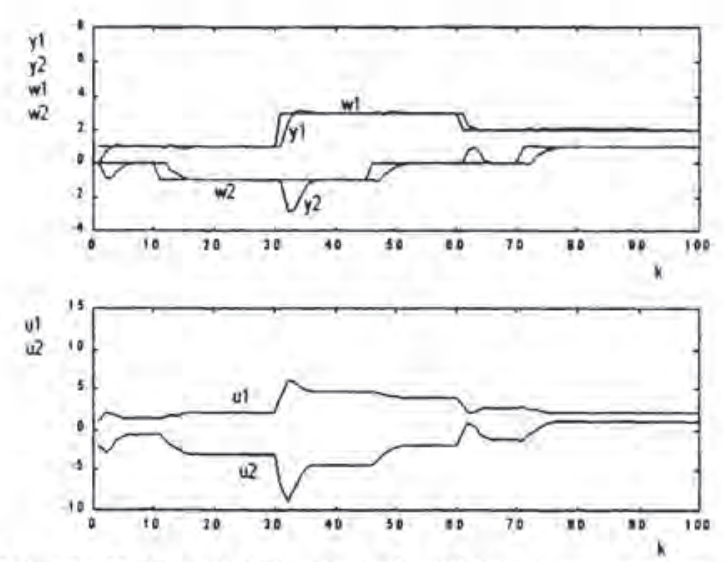

Fig. 7. Deterministic control without compensator
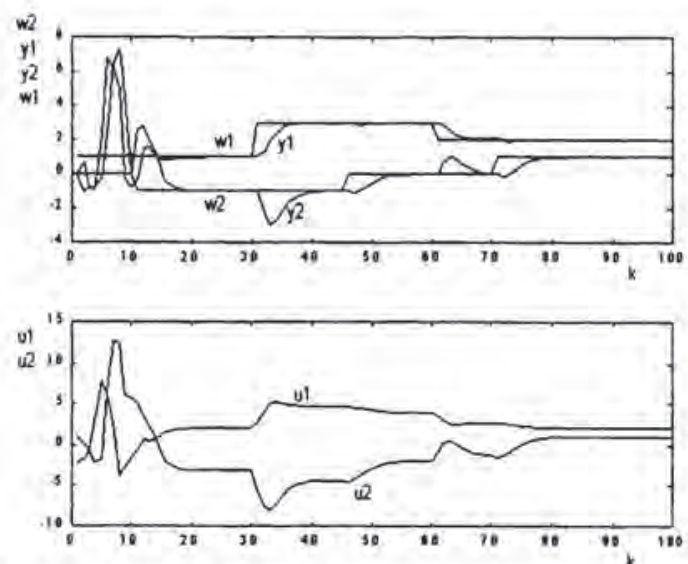

Fig. 8. Adaptive control without a compensator
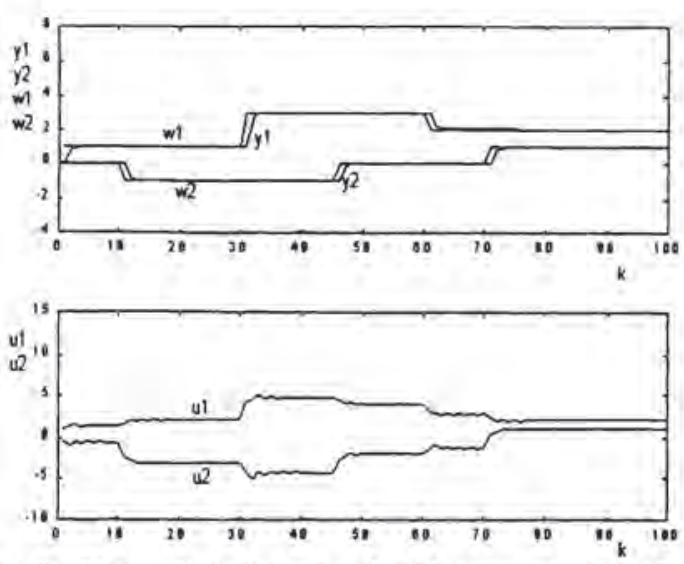

Fig. 9. Deterministic control with compensator $C_{1}$ 

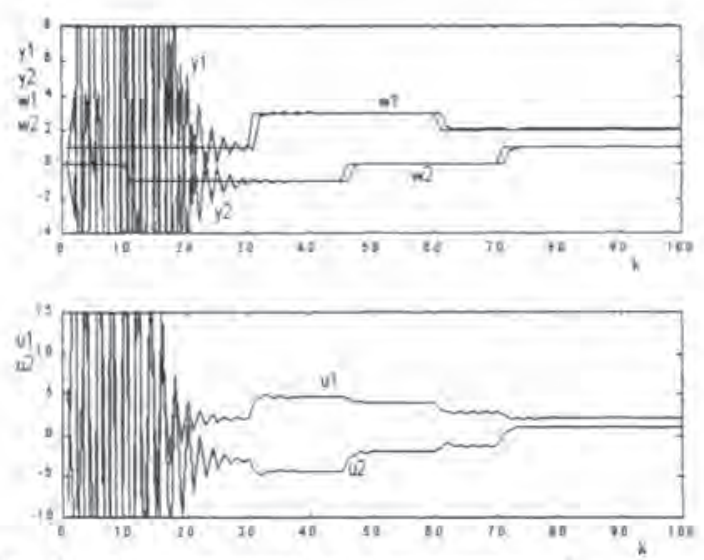

Fig. 10. Adaptive control with compensator $C_{1}$
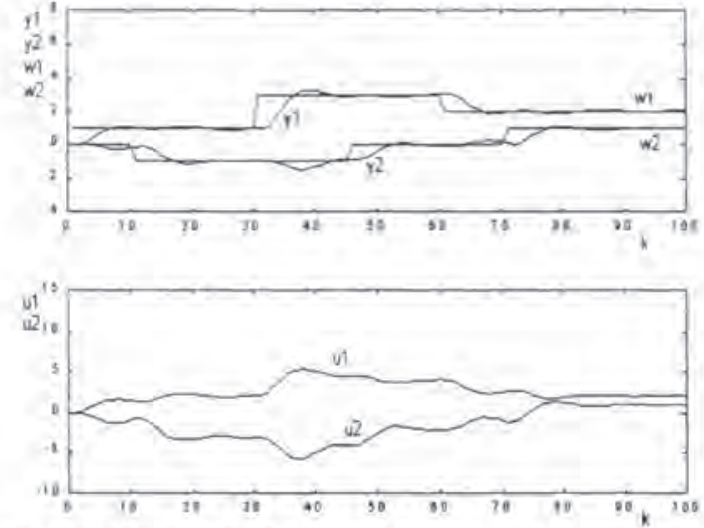

Fig. 11. Deterministic control with compensator $\mathbf{C}_{2}$
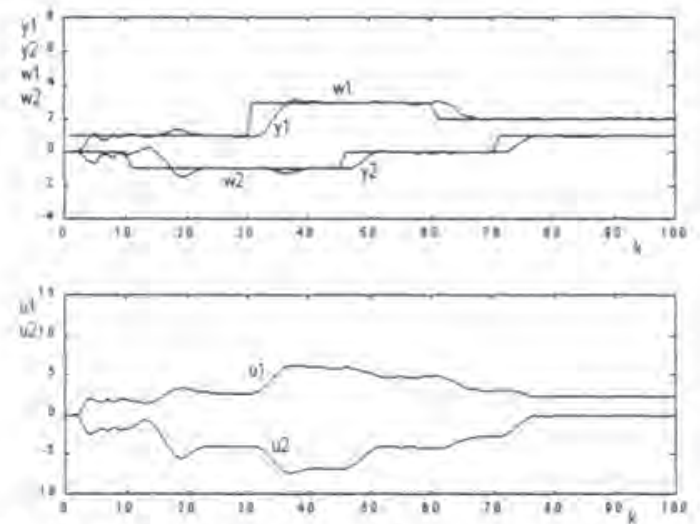

Fig. 12. Adaptive control with compensator $\mathbf{C}_{2}$

\section{VERIFICATION - CONTROLLING A LABORATORY MODEL}

Our department has experimental laboratory model CE 108 - couples drives apparatus. This apparatus, based on experience with authentic industrial control applications, was developed in cooperation with the University of Manchester and made by a British company, TecQuipment Ltd. It allows us to investigate the ever-present difficulty of controlling the tension and speed of material in a continuous process. The process may require the material speed and tension to be controlled to within defined limits. Examples of this occur in the paper-making industry, strip metal and wire manufacture and, indeed, any process where the product is manufactured in a continuous strip.

The industrial type material strip is replaced by a continuous flexible belt. The principle scheme of the model is shown in the Fig. (13). It consists of three pulleys, mounted on a vertical panel so that they form a triangle resting on its base. The two base pulleys are directly mounted on the shafts of two nominally identical servo motors and the apparatus is controlled by manipulating the drive torques to these servo motors. The third pulley, the jockey, is free to rotate and is mounted on a pivoted arm. The jockey pulley assembly, which simulates a material work station, is equipped with a special sensor and tension measuring equipment. It is the jockey pulley speed and tension which form the principle system outputs. The belt tension is measured indirectly by monitoring the angular deflection of the pivoted tension arm to which the jockey pulley is attached.

The manipulated variables are the inputs to the servo motors and the controlled variables are the tension and speed at the work station. There are interactions between the control loops.

The task was to apply the methods we designed for the adaptive control of a model representing a nonlinear system with variable parameters which is, therefore, impossible to control deterministically.

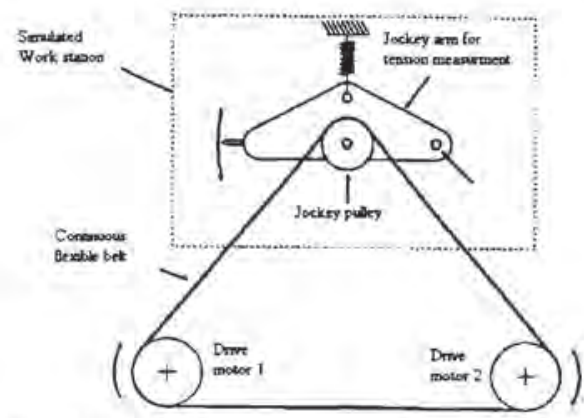

Fig. 13. Principal scheme of CE 108
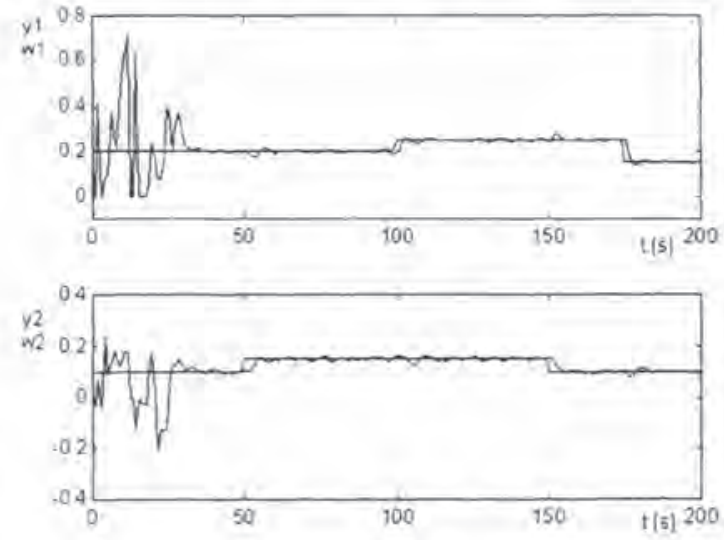

Fig. 14. The adaptive control of a real model without a compensator

Adaptive control using recursive identification both with and without the use of compensators was performed. As indicated in the simulation, compensator $\mathbf{C}_{\mathbf{1}}$ was shown to be unsuitable and 
control broke down. The other two methods gave satisfactory results. The time responses of the control for both cases are shown in Fig. 14, Fig. 15, Fig. 16 and Fig. 17. The figures demonstrate that control with a compensator reduces interaction. The controlled variable $y_{1}$ is the speed and the controlled variable $y_{2}$ is the tension.
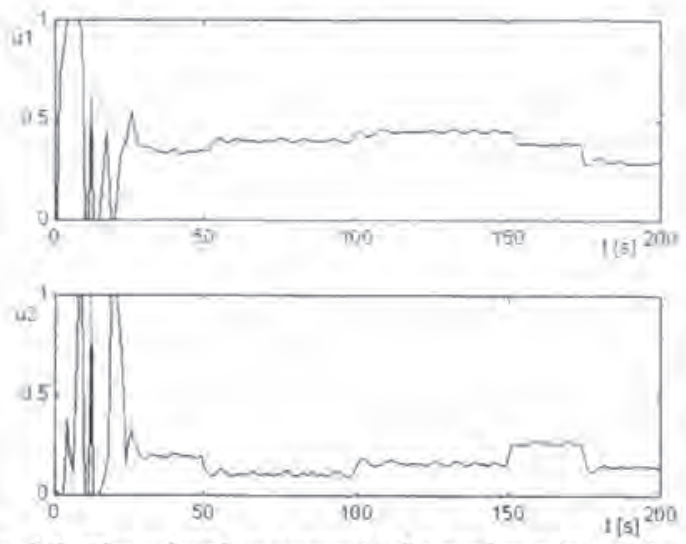

Fig. 15. The adaptive control of a real model without a compensator - controller output
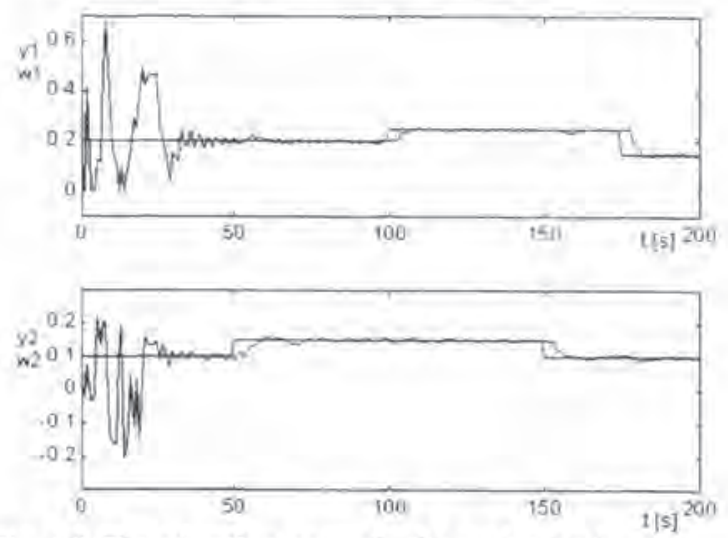

Fig. 16 The adaptive control of a real model using compensator $\mathbf{C}_{2}$
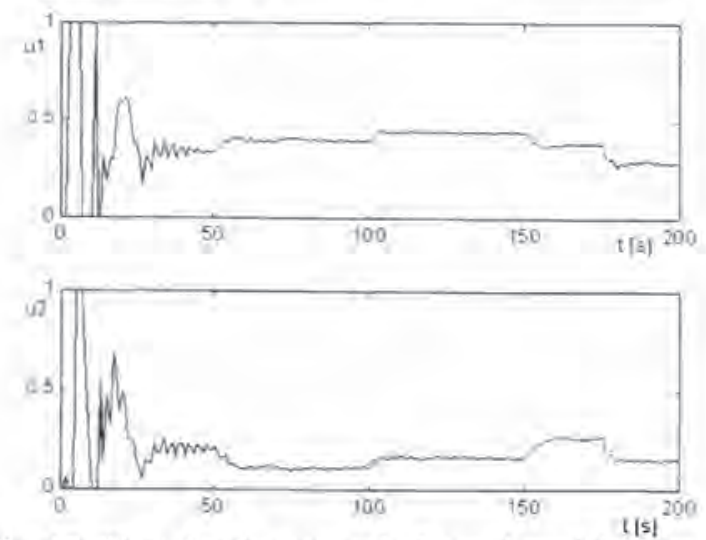

Fig. 17. The adaptive control of a real model using compensator $\mathbf{C}_{2}$ - controller output

\section{CONCLUSIONS}

The adaptive control of a two-variable system based on polynomial theory was designed. Decoupling problems were solved by the use of compensators.
The designs were simulated and used to control a laboratory model. The simulation results proved that these methods are suitable for the control of linear systems. The control tests on the laboratory model gave satisfactory results despite the fact that the nonlinear dynamics were described by a linear model.

\section{ACKNOWLEDGMENTS}

The work has been supported by the Grant Agency of the Czech Republic under grants No. 102/99/1292 and 102/01/P013 and by the Ministry of Education of the Czech Republic under grant No. MSM 281100001 . This support is very gratefully acknowledged.

\section{REFERENCES}

Bittanti, S., Bolzern, P., Campi, M. (1990). Convergence and Exponential Convergence of Identification Algorithms with Directional Forgetting Factor. Automatica, 26, 5, 929 - 932.

Chien, I.L., Seborg, D.E., Mellichamp, D. A. (1987). Self-Tuning Control with Decoupling. AIChE J., $33,7,1079-1088$

Krishnawamy, P.R. et al. (1991). Reference System Decoupling for Multivariable Control. Ind. Eng. Chem. Res., 30, 662-670.

Luyben W. L. (1986). Simple Method for Tuning SISO Controllers in Multivariable Systems. Ing. Eng. Chem. Process Des. Dev., 25, 654-660.

Peng, Y. (1990). A General Decoupling Precompensator for Linear Multivariable Systems with Application to Adaptive Control. IEEE Trans. Aut. Control, AC-35, 3, 344-348.

Skogestad, S., Postlethwaite, J. (1996) Multivariable Fedback Control - Analysis and Design. New York, J. Willey.

Tade, M.O., Bayoumi, M.M., Bacon, D.W. (1986). Adaptive Decoupling of a Class of Multivariable Dynamic Systems Using Output Feedback. IEE Proc. Pt.D, 133, 6, 265-275.

Wittenmark, B., Middelton, R. H., Goodwin, G.C. (1987). Adaptive Decoupling of Multivariable Systems. Int. J. Control, 46, 6, 1993-2009. 